\title{
Análise do perfil epidemiológico de adolescentes com sobrepeso e hipertensos no
}

\section{estado de Sergipe}

Analysis of the epidemiological profile of overweight and hypertensive adolescents in the state of

Sergipe

Análisis del perfil epidemiológico de adolescentes hipertensos y con sobrepeso en el estado de

Sergipe

\section{Resumo}

Introdução: A hipertensão arterial sistêmica (HAS) associada à sobrepeso e obesidade é uma condição muito comum no mundo, sua prevalência vem aumentando em crianças e adolescentes, sendo responsável por um grande impacto na qualidade de vida, com interferência direta no crescimento e desenvolvimento dos indivíduos. No Brasil, a prevalência hipertensão arterial sistêmica na população pediátrica varia de $1 \%$ a $13 \%$ e cerca de $20 \%$ dos adolescentes brasileiros estão com sobrepeso. A hipertensão arterial sistêmica na infância tem sido associada a recente epidemia de obesidade, crianças e adolescentes com níveis de pressão arterial elevados apresentam maior chance de se tornarem adultos portadores de hipertensão arterial sistêmica, que está associada a eventos como acidente vascular encefálico, infarto agudo do miocárdio, insuficiência cardíaca, doença arterial periférica e doença renal crônica. Justificativa: Há uma lacuna importante nas informações existentes acerca desse tema no estado de Sergipe, viabilizando, dessa forma, insuficiena construção desse projeto de pesquisa. Objetivo: Analisar o perfil epidemiológico e a associação entre a obesidade e hipertensão em adolescentes no estado de Sergipe. Métodos: Estudo epidemiológico descritivo, de dados secundários, de abordagem quantitativa, documental, transversa, exploratória, retrospectiva, de avaliação de saúde cujos dados foram coletados sistema de cadastramento e acompanhamento de hipertensos e diabéticos (HIPERDIA) DATASUS-MS, referente aos indivíduos diagnosticados com hipertensão arterial sistêmica em Sergipe no período de 2002 a 2013.

Palavras-chave: Adolescente; Hipertensão; Sobrepeso.

\begin{abstract}
Introduction: Systemic arterial hypertension $(\mathrm{SAH})$ associated with overweight and obesity is a very common condition in the world, its prevalence is increasing in children and adolescents, being responsible for a great impact on quality of life, with direct interference in the growth and development of individuals. In Brazil, the prevalence of systemic arterial hypertension in the pediatric population ranges from $1 \%$ to $13 \%$ and about $20 \%$ of Brazilian adolescents are overweight. Systemic arterial hypertension in childhood has been associated with a recent obesity epidemic, children and adolescents with high blood pressure levels are more likely to become adults with systemic arterial hypertension, which is associated with events such as stroke, acute myocardial infarction, heart failure, peripheral arterial disease and chronic kidney disease. Justification: There is an important gap in the existing information on this topic in the state of Sergipe, thus enabling the construction of this research project. Objective: To analyze the epidemiological profile and the association between obesity and hypertension in adolescents in the state of Sergipe. Methods: Descriptive epidemiological study of secondary data, quantitative, documentary, cross-sectional, exploratory, retrospective, health assessment approach whose data were collected in the registration and monitoring system for hypertensive and diabetic patients (HIPERDIA) - DATASUS-MS, referring to individuals diagnosed with systemic arterial hypertension in Sergipe from 2002 to 2013. Keywords: Adolescent; Hypertension; Overweight.
\end{abstract}




\begin{abstract}
Resumen
Introducción: La hipertensión arterial sistémica (HAS) asociada a sobrepeso y obesidad es una condición muy común en el mundo, su prevalencia está aumentando en niños y adolescentes, siendo responsable de un gran impacto en la calidad de vida, con interferencia directa en el crecimiento y desarrollo. de los individuos. En Brasil, la prevalencia de hipertensión arterial sistémica en la población pediátrica varía del 1\% al 13\% y alrededor del 20\% de los adolescentes brasileños tienen sobrepeso. La hipertensión arterial sistémica en la infancia se ha asociado con una epidemia de obesidad reciente, los niños y adolescentes con niveles de presión arterial alta tienen más probabilidades de convertirse en adultos con hipertensión arterial sistémica, que se asocia con eventos como accidente cerebrovascular, insuficiencia cardíaca, enfermedad arterial periférica y enfermedad renal crónica. Justificación: Existe un vacío importante en la información existente sobre este tema en el estado de Sergipe, lo que permite la construcción de este proyecto de investigación. Objetivo: Analizar el perfil epidemiológico y la asociación entre obesidad e hipertensión en adolescentes del estado de Sergipe. Métodos: Estudio epidemiológico descriptivo de datos secundarios, cuantitativo, documental, transversal, exploratorio, retrospectivo, enfoque de evaluación de la salud, cuyos datos fueron recolectados en el sistema de registro y seguimiento de pacientes hipertensos y diabéticos (HIPERDIA) - DATASUS-MS, referido a individuos. diagnosticado de hipertensión arterial sistémica en Sergipe de 2002 a 2013.
\end{abstract}

Palabras clave: Adolescente; Hipertensión; Sobrepeso.

\title{
1. Introdução
}

A Organização Mundial da Saúde (OMS) define a adolescência como a faixa etária de 10 - 19 anos, no mundo $20 \%$ da população estão nessa faixa etária, enquanto no Brasil são 17\%, totalizando 34 milhões de pessoas (Barbalho et al., 2020).

A adolescência é um período de transformações fisiológicas e psicossociais, que contribuem para a vulnerabilidade nutricional dessa população (Enes \& Slater, 2010).

A obesidade é considerada uma doença crônica, de etiologia multifatorial, que se desenvolve, na maioria das vezes devido a associação de fatores genéticos, metabólicos, nutricionais, psicossociais e ambientais. Os fatores mais associados a obesidade estão relacionados ao estilo de vida, principalmente mudanças nos padrões de comportamento alimentar e redução da atividade física. (Weffort, 2019).

É caracterizada por excesso de gordura corporal, e geralmente definida pelo índice de massa corporal (IMC), conseguido dividindo o peso corporal em quilogramas, pela altura em metros ao quadrado (Güngör, 2014)

Sua prevalência vem crescendo nas últimas décadas em todo o mundo, até mesmo nos países em desenvolvimento, como o Brasil, que há algumas décadas, predominavam problemas relacionados a desnutrição (Enes \& Slater, 2010).

De acordo com Neves et al (2020) a prevalência mundial de crianças e adolescentes com obesidade era de $1 \%$ em 1975 e subiu para $6 \%$ no ano 2016. Nos estados unidos 20,6\% dos adolescentes são obesos (Ruiz et al., 2019). No Brasil 20\% dos adolescentes estão com sobrepeso (Barbalho et al., 2020).

Alterações metabólicas, como baixos níveis de lipoproteínas de alta densidade (HDL-c) e altos níveis de triglicérides e glicose, geralmente estão associados a sobrepeso e obesidade (Mendes et al., 2019). A hipertensão arterial sistêmica (HAS) na infância tem sido associada a recente epidemia de obesidade (Figueirinha \& Herdy, 2017).

A HAS é definida por níveis de pressão arterial (PA) elevados de forma persistente, após 3 medidas sucessivas em que os níveis de PA estejam superiores ao percentil 95 para idade, sexo e estatura (Koch \& Silva, 2010).

A HAS pode ser caracterizada em primária, quando não se pode encontrar uma causa subjacente para a hipertensão, e secundaria, quando é possível identificar e tratar uma causa subjacente, atualmente nas crianças e adolescentes a hipertensão primária é responsável pela maior parte dos casos (Riley et al., 2018).

Segundo Moreira et al. (2013), no mundo são mais de 800 milhões de pessoas com hipertensão arterial. Nos Estados Unidos a prevalência da hipertensão arterial entre crianças e adolescentes varia de 0,3\% - 4,5\% (Rao, 2020). No Brasil 1\% $13 \%$ da população pediátrica é acometida pela hipertensão arterial (Koch \& Silva, 2010).

De acordo com Pinto e Silva (2015), crianças e adolescentes com níveis de PA elevados apresentam maior chance de 
se tornarem adultos portadores de HAS. A hipertensão arterial está associada a eventos como acidente vascular encefálico (AVE), infarto agudo do miocárdio (IAM), insuficiência cardíaca (IC), doença arterial periférica (DAP) e doença renal crônica (DRC) (Malachias et al., 2016). Moura et al. (2019) defendem que os estudos demonstram a associação entre obesidade e hipertensão, mas também relatam que os fatores de risco como baixos níveis de HDL-C, altos níveis de LDL-C e glicose sérica podem causar danos cardiovasculares de médio e longo prazo.

As doenças cardiovasculares são responsáveis por 30\% das mortes ocorridas no Brasil, sendo a principal causa de morte na população geral (Oliveira et al., 2020). De acordo com Santos et al. (2019), a HAS é a causa de grande número de mortes em adultos, por isso medidas preventivas podem ser tomadas desde a infância, evitando complicações futuras.

De acordo com Morais et al. (2011), as doenças cardiovasculares causam um grande impacto econômico, pois chegam a consumir até $12 \%$ das verbas de um hospital público geral.

Desse modo, se faz necessário o diagnóstico precoce e prevenção dos fatores de risco durante a infância e adolescência, visando evitar futuras complicações cardiovasculares (Medeiros et al., 2012). São necessário novos estudos sobre a obesidade infanto-juvenil, visando novas formas de intervenção para a promoção de saúde dessa população (Teles et al., 2012).

\section{Metodologia}

O presente trabalho obedeceu a todas as normas éticas emanadas da resolução 466/12, no que se refere à pesquisa envolvendo seres humanos. Como o estudo foi baseado em dados populacionais, não houve necessidade de envio do projeto à Plataforma Brasil.

O estudo foi realizado no município de Lagarto, abrangendo todas as pessoas que foram diagnosticadas com hipertensão arterial sistêmica e em acompanhamento do estado nutricional, na faixa etária dos 10 aos 19 anos, no período de 2002 a 2013 registrados no sistema de cadastramento e acompanhamento de hipertensos e diabéticos (HIPERDIA) do DATASUS.

A referida pesquisa tratou-se de um estudo epidemiológico, descritivo de dados secundários, de abordagem quantitativa, documental, transversa, de avaliação de saúde.

De acordo com Hamann e Tauil (2021) as pesquisas epidemiológicas descritivas estudam a ocorrência e distribuição de eventos, visando verificar ou não a associação entre variáveis de exposição e efeito.

Segundo Gil (2008) um dos objetivos de uma pesquisa descritiva pode ser estudar as características de um grupo, como sua distribuição por sexo, idade, procedência, período, fatores de risco, etc.

O método quantitativo é caracterizado pelo uso da quantificação, seja na coleta de informações ou mesmo na análise dos dados, podendo ser usadas técnicas estatísticas, como percentual, média, desvio-padrão, etc. É um método bastante utilizado em estudos descritivos (Richardson et al., 1999).

Um estudo documental utiliza dados que não receberam um tratamento analítico, ou que podem ser utilizados de acordo com os objetivos da pesquisa, esses dados podem ser obtidos de relatórios de pesquisa, relatórios de empresas, tabelas estatísticas etc. (Gil, 1999).

De acordo com Richardson (1999) em um estudo transversal os dados são obtidos em um determinado momento do tempo e visam analisar ou descrever informações existentes no momento da coleta de dados.

O estudo foi do tipo epidemiológico descritivo, onde foram incluídos todos os casos de hipertensão arterial sistêmica e pessoas em acompanhamento do estado nutricional, na faixa etária dos 10 aos 19 anos, registrados no estado de Sergipe, no período de 2002 a 2013, cadastrados no sistema de cadastramento e acompanhamento de hipertensos e diabéticos (HIPERDIA) - DATASUS-MS. perfazendo um total de 347 casos.

A coleta de dados foi realizada no sistema de cadastramento e acompanhamento de hipertensos e diabéticos 
(HIPERDIA) - DATASUS-MS, através de tabelas emitidas pelo sistema, de acordo com as seguintes variáveis de interesse de estudo frequências absolutas e relativas com proporção na base 100.

Os resultados foram mostrados através de tabelas e gráficos para melhor compreensão.

Foi utilizado como critério de inclusão pacientes que tiveram hipertensão arterial sistêmica e sobrepeso ou obesidade no período de estudo, na faixa etária dos 10 aos 19 anos e estavam registrados no sistema de cadastramento e acompanhamento de hipertensos e diabéticos (HIPERDIA) - DATASUS-MS. Como critério de exclusão foi utilizado ter sido diagnosticado com hipertensão arterial sistêmica e não ser residente do estado do Sergipe, e estar fora da faixa etária alvo do estudo.

Foram analisadas as seguintes variáveis: dados sociodemográficos (sexo e faixa etária), fatores de risco (obesidade e sedentarismo), região de saúde e período.

O estudo trouxe risco mínimo aos participantes, uma vez que foram utilizados dados populacionais e bancos de dados epidemiológicos, não havendo, portanto, risco de identificação dos participantes. Mesmo assim, todas as normas da resolução 466/12 foram atendidas no sentido de minimizar esse risco. O conhecimento do perfil epidemiológico desses pacientes trará como benefício a possibilidade de se adotar medidas para combater o aparecimento de novos casos da doença.

\section{Resultados e Discussão}

Em Sergipe, entre os anos de 2002 a 2013, de acordo com o DATASUS, através do sistema de cadastramento e acompanhamento de hipertensos e diabéticos (HIPERDIA), o número de hipertensos diagnosticados na faixa etária dos 10 aos 19 anos totalizaram 347 casos, com relação ao sexo dos participantes, os dados mostraram maior frequência no sexo feminino, representando 65,41\% (n=227) dos casos, enquanto o sexo masculino representa 34,59\% dos casos (n=120) (figura 1).

Os resultados do presente estudo não corroboram com os dados encontrados por Bloch et al. (2015), em um estudo seccional, nacional, de base escolar, onde a prevalência de hipertensão arterial sistêmica foi maior nos adolescentes do sexo masculinos. Resultados semelhantes foram encontrados por Figueirinha e Herdy (2017), onde 76,5\% dos adolescentes participantes que apresentaram pressão arterial alterada pertenciam ao sexo masculino. Uma revisão sistemática e metanálise de Gonçalves et al. (2016), também encontrou uma prevalência maior em adolescentes do sexo masculino.

Os jovens do sexo masculino são menos propensos a procurar o serviço de saúde em comparação com as jovens do sexo feminino (Dasgupta et al., 2006). Uma possibilidade para explicar a divergência com a literatura encontrada no presente estudo, seria a baixa adesão aos serviços de saúde evidenciada em indivíduos do sexo masculino, logo que as informações foram obtidas do DATASUS, através de dados coletados no HIPERDIA, que é um sistema de cadastramento e acompanhamento de hipertensos e diabéticos atendidos na rede ambulatorial do sistema único de saúde - SUS. 
Figura 1: -Hipertensão no estado de Sergipe, na faixa etária dos 10 aos 19 anos, por sexo, no período de 2002 a 2013.

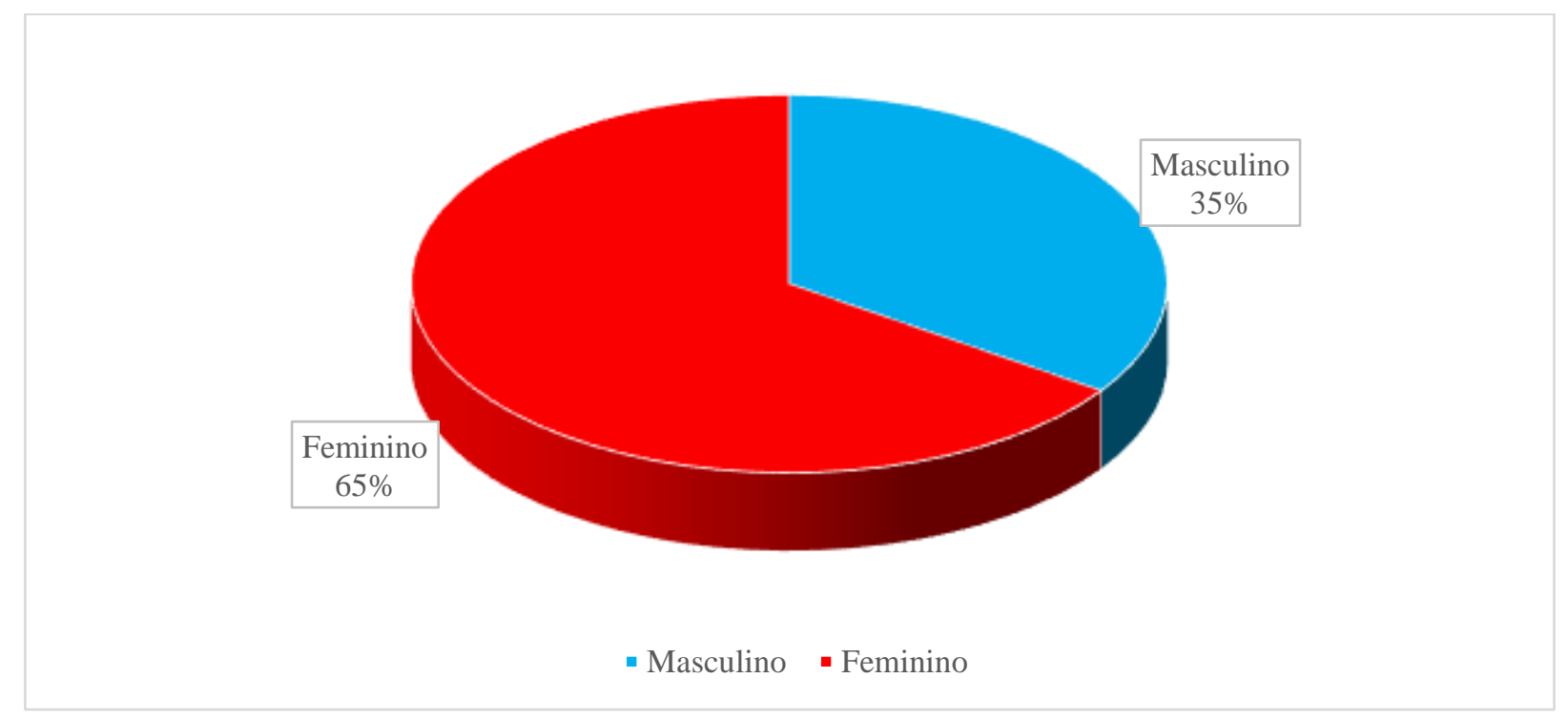

Fonte: DATASUS (2021).

Dos 347 participantes do estudo, 26,22\% ( $\mathrm{n}=91$ ) apresentavam sobrepeso. A porcentagem de meninas com sobrepeso foi maior quando comparada aos meninos, $74,72 \%(\mathrm{n}=68)$ dos indivíduos que apresentaram sobrepeso eram do sexo feminino, enquanto $25,28 \%$ ( $n=23$ ) eram do sexo masculino (figura 2).

Lamounier e Abrantes (2003) em sua revisão de literatura sobre a prevalência de obesidade e sobrepeso na adolescência no Brasil, evidenciaram que a prevalência de sobrepeso e obesidade variou entre 7,8\% a 22,3\%, entretanto na maioria dos estudos a prevalência foi maior no sexo masculino, quando comparado ao sexo feminino.

Em Curitiba, a prevalência no sexo masculino foi $11,2 \%$ e, no feminino, 4,4\%, em Florianópolis houve maior incidência de obesidade no sexo masculino (14,6\%), comparado ao sexo feminino (7,7\%). Em Recife o sobrepeso foi maior nos adolescentes do sexo masculino $(35,7 \%)$ do que nos do sexo feminino (11,2\%). Em São Paulo a prevalência de sobrepeso foi de 15,6\% e $14,0 \%$ de sobrepeso para os sexos masculino e feminino, respectivamente. No Rio de Janeiro a prevalência de sobrepeso foi de $28,6 \%$ para o sexo masculino e 15,5\% para o feminino (Lamounier \& Abrantes, 2003).

Tais discrepâncias entre os resultados desse estudo e a literatura, provavelmente se devem a baixa utilização dos adolescentes do sexo masculino aos serviços da rede ambulatorial do sistema único de saúde, resultando em uma amostra majoritariamente feminina. 
Figura 2: Hipertensão associado a sobrepeso no estado de Sergipe, na faixa etária dos 10 aos 19 anos, por sexo, no período de 2002 a 2013.

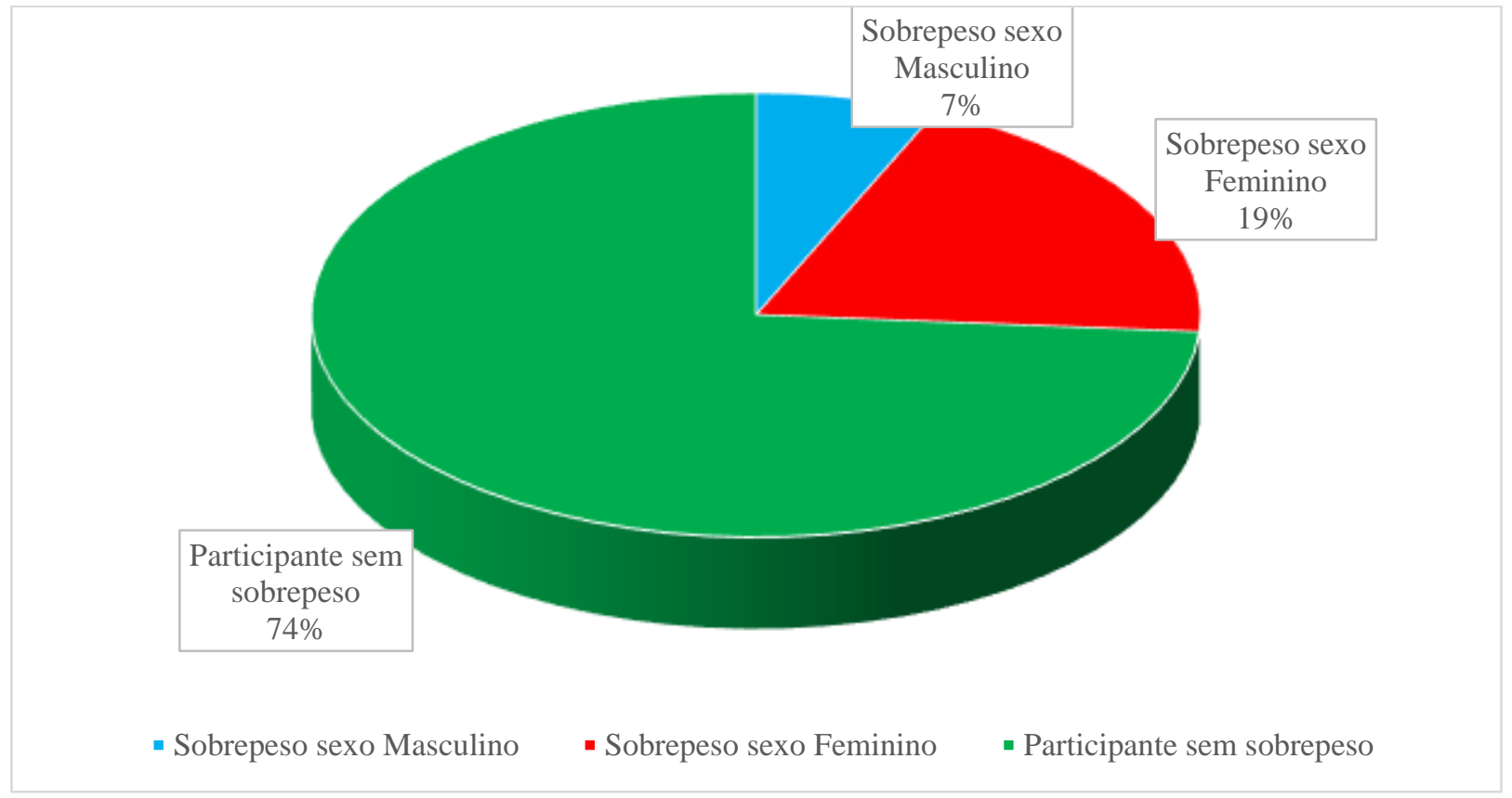

Fonte: DATASUS (2021).

38,32\% ( $\mathrm{n}=133)$ dos participantes do estudo eram sedentários, deste total 30,83\% ( $\mathrm{n}=41)$ eram do sexo masculino, enquanto 69,17\% (n=92) eram do sexo feminino (figura 3). Tais resultados obtidos no presente estudos são corroborados por dados encontrados na literatura.

Piola et al. (2020), em seu estudo sobre fatores associados ao nível insuficiente de atividade física e elevado tempo de tela em adolescentes, evidenciou que as meninas são 15,97\% mais propensas que os meninos a não cumprirem as recomendações mínimas de atividade física. Stochero et al. (2018) associaram o sedentarismo positivamente ao sexo feminino em seu estudo com adolescentes estudantes da rede de ensino pública e privada, do $8^{\circ}$ ano do ensino fundamental ao $3^{\circ}$ ano do ensino médio. Alves e Silva (2013), em seu artigo de revisão, encontraram a prevalência de sedentarismo variando entre 5,3\% a 49,7\%; no sexo masculino e $15 \%$ a $74,1 \%$ no sexo feminino. 
Figura 3: Hipertensão associado a sedentarismo no estado de Sergipe, na faixa etária dos 10 aos 19 anos, por sexo, no período de 2002 a 2013

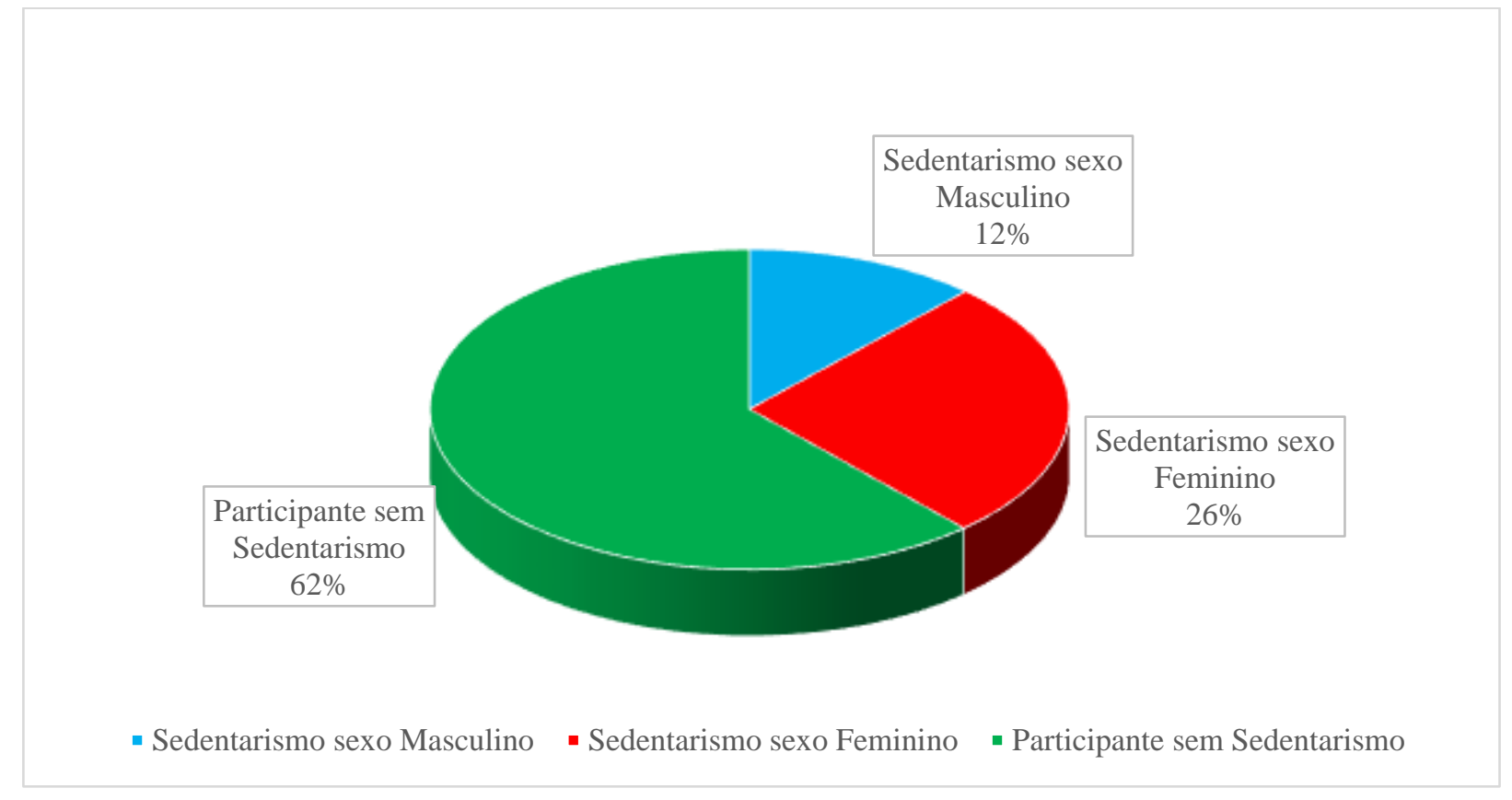

Fonte: DATASUS (2021).

14,99\% (n=52) dos participantes apresentaram sedentarismo e sobrepeso associado à hipertensão. Quase metade dos participantes 49,57\% $(\mathrm{n}=172)$ da amostra apresentaram sedentarismo, obesidade ou ambos os fatores de risco para hipertensão arterial sistêmica (figura 4).

Figueirinha e Herdy (2017) em um estudo observacional de corte transversal, na cidade de Petrópolis-RJ, com 157 adolescentes, encontraram IMC alterado em 31,21\% $(n=49)$ da população estudada, entre os hipertensos a prevalência de IMC alterado foi de $70,58 \%(n=12)$.

Guedes e Mello (2021) em recente metanálise encontrou prevalência de obesidade variando entre 11,4\% e 27,2\%, e $9,5 \%$ e $26,9 \%$ em adolescentes do sexo feminino e masculino, respectivamente. As taxas mais elevadas foram encontradas em estudos realizados na região sul.

Os resultados do presente estudo em relação aos fatores de risco sobrepeso e sedentarismo são condizentes com os dados encontrados na literatura, quando analisados de maneira global, as divergências são maiores quando comparados em relação ao sexo dos participantes. 
Figura 4: Hipertensão associado a sedentarismo ou sobrepeso, no estado de Sergipe, na faixa etária dos 10 aos 19 anos, no período de 2002 a 2013.

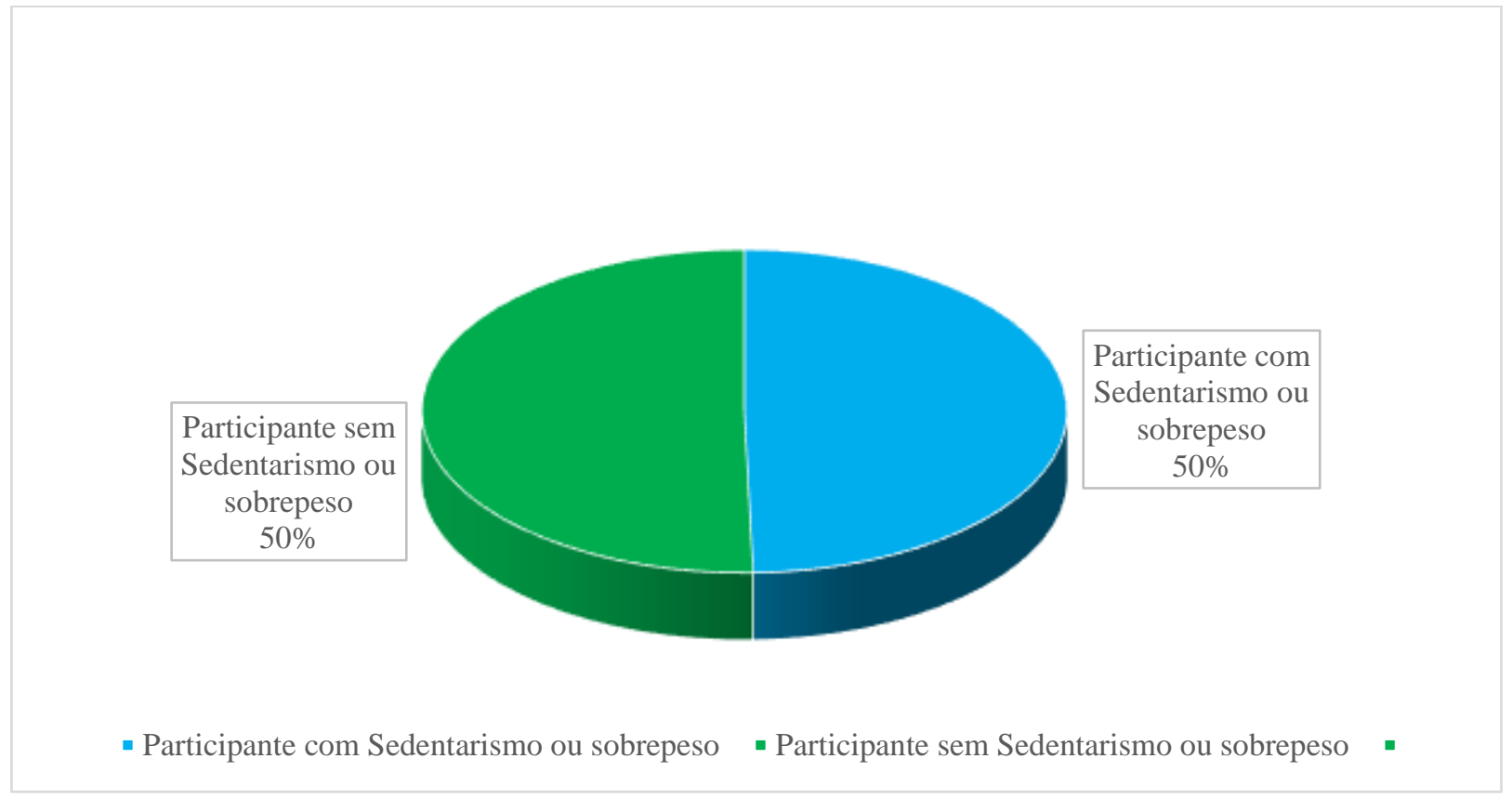

Fonte: DATASUS (2021).

Em relação a distribuição dos casos, de acordo com as regiões de saúde do estado de Sergipe, verificou-se o predomínio de casos nas regiões de Nossa Senhora do Socorro 26,22\% (n=91), Estância 16,14\% (n=56), Lagarto 15,85\% $(\mathrm{n}=55)$, Itabaiana $15,27 \%(\mathrm{n}=53)$ e Propriá $13,83 \%(\mathrm{n}=48)$ (Tabela 1$)$.

Os resultados obtidos, em relação à distribuição dos casos, chamam atenção devido a desproporcionalidade da frequência de casos de hipertensão arterial sistêmica em relação ao total de habitantes da região em saúde Aracaju.

De acordo com dados do Instituto Brasileiro de Geografia e estatística (IBGE) (2012), a cidade de Aracaju possuía 25,86\% $(n=461.534)$ dos habitantes do estado de Sergipe, no presente estudo, apenas 7,20\% ( $n=25)$ da amostra foi proveniente da região de saúde de Aracaju, indicando grande desproporcionalidade, quando comparado as outras regiões de saúde do estado.

Essa desproporcionalidade pode ser causada por subdiagnóstico, subnotificação ou ambos.

É muito comum que a hipertensão arterial sistêmica se apresente em sua forma assintomática, desse modo, muitas pessoas não conhecem sua condição de saúde, resultando em uma grande quantidade de falsos negativos (Julião \& Guimarães, 2013).

O subdiagnóstico de hipertensão arterial sistêmica em adolescentes é comum, o principal fator causal é a necessidade de utilização de tabelas de percentis, que exigem maior tempo, sendo assim, geralmente deixada de lado devido a relativa baixa prevalência de hipertensão arterial sistêmica nos adolescentes (Mourato, 2014).

Em seu estudo sobre subnotificação, Giroto et al (2010) encontraram a taxa de 39,5\% de subnotificações no HIPERDIA, Os principais motivos de subnotificação são não residir mais na área da USF (36,7\%), não ser cadastrado na respectiva fonte $(29,3 \%)$ e fazer controle da pressão arterial em outro serviço $(24,0 \%)$. 
Tabela 1: Hipertensão segundo Região de Saúde (CIR), no estado de Sergipe, na faixa etária dos 10 aos 19 anos, no período de 2002 a 2013.

\begin{tabular}{|c|c|}
\hline Região de Saúde (CIR) & Hipertensão \\
\hline TOTAL & 347 \\
\hline Aracaju & 25 \\
\hline Estância & 56 \\
\hline Itabaiana & 53 \\
\hline Lagarto & 19 \\
\hline Nossa Senhora da Glória & 91 \\
\hline Nossa Senhora do Socorro & 48 \\
\hline Propriá & \\
\hline
\end{tabular}

Fonte: DATASUS (2021).

No presente estudo, a análise temporal de 2002 a 2013, mostrou que o número de pessoas na faixa etária dos 10 aos 19 anos diagnosticados com hipertensão arterial sistêmica variou bastante. Os anos que registraram o maior número de casos foram 2002 e 2006, com 19,02\% (n=66) e 17,86\% (n=62), respectivamente. Os anos com menor número de casos foram 2003, 2004 e $2013 \mathrm{com} 4,61 \%(\mathrm{n}=16), 3,17 \%(\mathrm{n}=11)$ e $0,57 \%(\mathrm{n}=2)$ respectivamente, vale ressaltar que só foram analisados os meses de janeiro a abril de 2013, por disponibilidade no sistema. (figura 5).

Em números absolutos a frequência de casos de hipertensão arterial sistêmica diminuiu de 66 casos em 2002 para 22 casos no ano de 2012, último ano com registro completo no sistema de cadastramento e acompanhamento de hipertensos e diabéticos (HIPERDIA). Resultados que divergem da literatura.

De acordo com Malachias et al. (2016), sugere-se que o número de adolescente com diagnostico de hipertensão arterial sistêmica dobrou na última década, provavelmente devido ao aumento da prevalência de obesidade infantil.

As divergências na literatura provavelmente se devem a subdiagnóstico ou subnotificação. 
Figura 5: Numero de hipertensos diagnosticados e acompanhados de 2002 a 2013, na faixa etária dos 10 aos 19 anos, no estado de Sergipe.

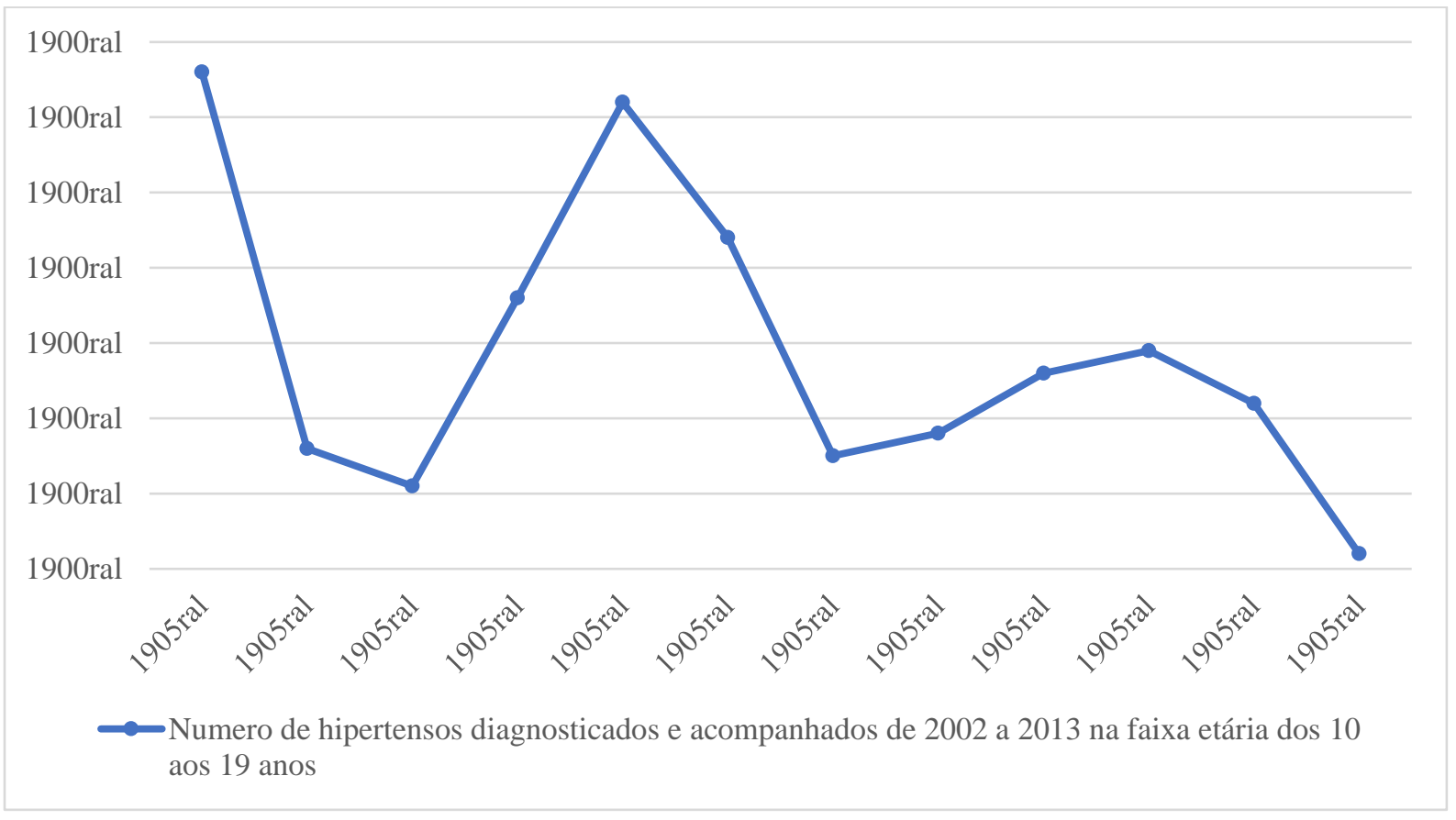

Fonte: DATASUS (2021).

\section{Conclusão}

O presente estudo possibilitou concluir que a maioria dos adolescentes com hipertensão, diagnosticadas e acompanhadas no estado de Sergipe, são do sexo feminino, resultados que divergem da maioria dos estudos sobre hipertensão nessa faixa etária, que geralmente encontra a prevalência maior no sexo masculino. Nos adolescentes hipertensos e com sobrepeso a frequência foi maior em adolescentes do sexo feminino, resultados que também se contrapõem a literatura, onde a maioria dos casos ocorrem em adolescentes do sexo masculino. Essas divergências de resultados provavelmente ocorrem devido baixa adesão aos serviços de saúde em adolescentes do sexo masculino.

Os resultados obtidos, em relação à distribuição dos casos, foram desproporcionais, a capital Aracaju, a maior cidade do estado e com maior número de habitantes, registrou menos casos do que cidades menores como Lagarto e Estância. A análise temporal mostrou que o número de casos diagnosticados diminuiu entre os anos de 2002 e 2013, divergindo da literatura, onde os estudos encontram aumento da prevalência de hipertensão nos adolescentes nas últimas décadas. Tais desproporcionalidade e divergências com a literatura podem ser causadas por subnotificação ou subdiagnóstico.

Existem poucos estudos sobre hipertensão e obesidade em adolescentes no estado de Sergipe, resultando em dificuldade na realização do estudo no sentido de encontrar dados na literatura sobre adolescentes hipertensos.

Diante do exposto, o conhecimento do perfil epidemiológico de adolescentes com sobrepeso e hipertensos no estado de Sergipe, é essencial para que os órgãos de saúde possam formular políticas públicas de saúde direcionadas a população estudada. Desse modo, recomenda-se a realização de mais estudos para investigar as possíveis causas das divergências com a literatura obtidas no atual estudo. 


\section{Referências}

Alves, C. F. D. A., \& Silva, R. D. C. R. (2013). Fatores associados à inatividade física em adolescentes: um artigo de revisão. Práticas investigativas em atividade física e saúde, 129-151. http://books.scielo.org

Barbalho, E. D. V., Pinto, F. J. M., Silva, F. R. D., Sampaio, R. M. M., \& Dantas, D. S. G. (2020). Influência do consumo alimentar e da prática de atividade física na prevalência do sobrepeso/obesidade em adolescentes escolares. Cad Saúde Colet, 1(28), 12-23. https://doi.org/10.1590/1414-462X202028010181v

Bloch, K. V., Klein, C. H., Szklo, M., Kuschnir, M. C. C., Abreu, G. D. A., Barufaldi, L. A., Veiga, G. V. D., Schaan, B., \& Silva, T. L. N. D. (2016). ERICA: prevalências de hipertensão arterial e obesidade em adolescentes brasileiros. Rev Saúde Pública, (50). https://doi.org/DOI:10.1590/S015188787.2016050006685

Dasgupta, K., O’Loughlin, J., Chen, S., Karp, I., Paradis, G., Tremblay, J., Hamet, P., \& Pilote, L. (2006). Emergence of Sex Differences in Prevalence of High Systolic Blood Pressure Analysis of a Longitudinal Adolescent Cohort. Circulation AHA. https://doi.org/DOI: 10.1161/CIRCULATIONAHA.106.624536

Enes, C. C., \& Slater, B. (2010). Obesidade na adolescência e seus principais fatores determinantes. Rev Bras Epidemiol, 1(13), $163-171$.

Figueirinha, F., \& Herdy, G. V. H. (2017). Hipertensão Arterial em Pré-Adolescentes e Adolescentes de Petrópolis: Prevalência e Correlação com Sobrepeso e Obesidade. Int J Cardiovasc Sci, 30(3), 243-250. https://doi.org/DOI: 10.5935/2359-4802.20170040

Gil, A. C. (2008). Métodos e técnicas de pesquisa social. (6th ed.). Atlas.

Girotto, E., Andrade, S. M. D., \& Cabrera, M. A. S. (2010). Análise de três fontes de informação da atenção básica para o monitoramento da hipertensão arterial. Epidemiol. Serv. Saúde, 19(2), 133-141.

Gonçalves, V. S. S., Galvão, T. F., Andrade, K. R. C. D., Dutra, E. S., Bertolin, M. N. T., Carvalho, K. M. B. D., \& Pereira, M. G. (2016). Prevalência de hipertensão arterial entre adolescentes: revisão sistemática e metanálise. Rev Saúde Pública. https://doi.org/DOI:10.1590/S1518-8787.2016050006236

Guedes, D. P. \& Mello, E. R. B. (2021). Prevalência de sobrepeso e obesidade em crianças e adolescentes brasileiros: Revisão sistemática e metanálise. ABCS Health SCI. 46:E021301

Güngör, N. K. (2014). Overweight and Obesity in Children and Adolescents. J Clin Res Pediatr Endocrinol, 6(03), 129-143. https://doi.org/DOI: $10.4274 /$ jcrpe. 1471

Hamann, E. M., \& Tauil, P. L. (2021). Proposta de classificação dos diferentes tipos de estudos epidemiológicos descritivos. Epidemiol. Serv. Saude, 30(1). https://doi.org/doi: 10.1590/S1679-49742021000100026

Instituto brasileiro de geografia e estatística (IBGE) (2012). Censo brasileiro de 2010.

Julião, N., \& Guimarães, R. (2018). Fatores associados ao subdiagnóstico de hipertensão na população brasileira: Um estudo com base na Pesquisa Nacional de Saúde (2013). Anais do XXI Encontro Nacional de Estudos Populacionais.

Koch, V. H. K., \& Silva, A. C. S. (2010). Tratado de pediatria: Sociedade Brasileira de Pediatria (2nd ed.). Manole.

Lamounier, J. A., \& Abrantes, M. M. (2003). Prevalência de obesidade e sobrepeso na adolescência no Brasil. Rev Med Minas Gerais, 13(4), 275-84.

Malachias, M. V. B., Souza, W. K. S. B., Plavnik, F. L., Rodrigues, C. I. S., Brandão, A. A., Neves, M. F. T., Bortolotto, L. A., Franco, R. J. S., Poli-deFigueiredo, C. E., Jardim, P. C. B. V., Amodeo, C., Barbosa, E. C. D., Koch, V., Gomes, M. A. M., Paula, R. B., Póvoa, R. M. S., Colombo, F. C., Ferreira, F. S., Miranda, R. D., Machado, C. A., Nobre, F., Nogueira, A. R., Mion, J. D., Kaiser, S., Forjaz, C. L. M., Almeida, F. A., Martim, J. F. V., Sass, N., Drager, L. F., Muxfeldt, E., Bodanese, L. C., Feitosa, A. D., Malta, D., Fuchs, S., Magalhães, M. E., Oigman, W., Moreira, F. O., Pierin, A. M. G., Feitosa, G. S., Bortolotto, M. R. F. L., Magalhães, L. B. N. C., Silva, A. C. S., Ribeiro, J. M., Borelli, F. A. O., Gus, M., Passarelli, J. O., Toledo, J. Y., Salles, G. F., Martins, L. C., Jardim, T. S. V., Guimarães, I. C. B., Antonello, I. C., Lima, J. E., Matsudo, V., Silva, G. V., Costa, L. S., Alessi, A., Scala, L. C. N., Coelho, E. B., Souza, D., Lopes, H. F., Gowdak, M. M. G., Cordeiro, J. A. C., Torloni, M. R., Klein, M. R. S. T., Nogueira, P. K., Lotaif, L. A. D., Rosito, G. B. A., \& Moreno, J. H. (2016). 7 Diretriz Brasileira de Hipertensão Arterial. Sociedade Brasileira de Cardiologia, 107(3).

Medeiros, C. C. M., Xavier, I. S., Santos, V. E. F. A., Souza, M. A. d. O., Vasconcelos, A. S. d., \& Alves, E. R. P. (2012). Obesidade infantil como fator de risco para a hipertensão arterial: uma revisão integrativa. Rev. Min. Enferm, 16(1), 111-119.

Mendes, M. G., Nascimento, L. M., Gomes, K. R. O., Araújo, R. S. D. R. M., Rodrigues, M. T. P., Araújo, T. M. E. D., \& Frota, K. D. M. G. (2019). Prevalência de Síndrome Metabólica e associação com estado nutricional em adolescentes. Cad. Saúde Colet, 27(4), 374-379. https://doi.org/DOI: 10.1590/1414462X201900040066

Morais, M. G. T., Pontes, W. C., \& Martins, A. S. (2011, November 7). Impacto das doenças cardiovasculares no serviço público. XVIII Congresso Brasileiro de Custos, Rio de Janeiro - RJ, Brasil.

Moreira, N. F., Muraro, A. P., Barbosa, F. d. S. B., Silva, R. M. V. G., Sichieri, R., \& Ferreira, M. G. (2013). Obesidade: principal fator de risco para hipertensão arterial sistêmica em adolescentes brasileiros participantes de um estudo de coorte. Arq Bras Endocrinol Metab, 57(7), 520-6.

Moura, C. M., Cabral, D., Tavares, G. C., Mendes, L. C., Figueira, L. A. F., \& Nunes, C. P. (2019). associação entre a incidência de hipertensão e obesidade em adolescentes. Revista Caderno de Medicina, 2(1).

Mourato, F. A. (2014). Auxilio na identificação de pressão arterial elevada em crianças através de uma modificação da razão entre pressão arterial e altura [Doctoral dissertation, Universidade Federal de Pernambuco]. 
Research, Society and Development, v. 10, n. 15, e104101522521, 2021

(CC BY 4.0) | ISSN 2525-3409 | DOI: http://dx.doi.org/10.33448/rsd-v10i15.22521

Neves, S. C., Rodrigues, L. M., Bento, P. A. d. S. S., \& Minayo, M. C. d. S. (220). Os fatores de risco envolvidos na obesidade no adolescente: Uma revisão integrativa. Cien Saude Colet. http://www.cienciaesaudecoletiva.com.br/artigos/os-fatores-de-risco-envolvidos-na-obesidade-no-adolescente-uma-revisaointegrativa/17534? id=17534

Oliveira, G. M. M. d., Brant, L. C. C., Polanczyk, C. A., Biolo, A., Nascimento, B. R., Malta, D. C., Souza, M. d. F. M. d., Soares, G. P., Junior, G. F. X., Machline-Carrion, M. J., Bittencourt, M. S., Pontes-Neto, O. M., Silvestre, O. M., Teixeira, R. A., Sampaio, R. O., Gaziano, T. A., Roth, G. A., \& Ribeiro, A. L. P. (2020). Estatística Cardiovascular - Brasil 2020. SBC. https://doi.org/DOI: https://doi.org/10.36660/abc.20200812

Pinto, S. L., \& Silv, R. d. C. R. (2015). Hipertensão arterial na infância e adolescência - prevalência no Brasil e fatores associados: uma revisão. Rev. Ciênc. Méd. Biol, 14(2), 225-232.

Piola, T. S., Bacil, E. D. A., Pacífico, A. B., Camargo, E. M. d., \& Campos, W. d. (2020). Nível insuficiente de atividade física e elevado tempo de tela em adolescentes: impacto de fatores associados. Ciência \& Saúde Coletiva, 25(7), 2803-2812. https://doi.org/DOI: 10.1590/1413-81232020257.24852018

Rao, G. (2016). Diagnosis, Epidemiology, and Management of Hypertension in Children. Pediatrics, 138(2). https://doi.org/DOI: 10.1542/peds.2015-3616

Richardson, R. J. (2012). Pesquisa social: Métodos e técnicas (3rd ed.). Atlas.

Riley, M., Hernandez, A. K., \& Kuznia, A. L. (2018). High Blood Pressure in Children and Adolescents. American Family Physician, 98(8).

Ruiz, L. D., Zuelch, M. L., Dimitratos, S. M., \& Scherr, R. E. (2020). Adolescent Obesity: Diet Quality, Psychosocial Health, and Cardiometabolic Risk Factors. Nutrients, 12(43). https://doi.org/doi:10.3390/nu12010043

Santos, R. d. J. L. d. L., Sousa, E. P. d., Rodrigues, G. M. d. M., Alexandre, K. V., Rabelo, L. M., \& Quaresma, P. d. C. (2019). Hipertensão Arterial Sistêmica Em Crianças E Adolescentes -Causas E Profilaxias. Braz. J. Hea. Rev, 2(2), 1063-1069.

Stochero, N., Leal, G. V. d. S., Ceni, G. C., \& Kirsten, V. R. (2018). Atividade física e fatores associados em adolescentes do interior do Rio Grande do Sul. Saúde (Santa Maria), 44(1), 1-9.

Teles, J. A., Costa, K. M., Lautner, M. A. F. A., \& Lautner, R. Q. (2012). Índice de prevalência de obesidade e hipertensão em crianças e pré adolescentes no ensino público de Formiga MG. Rev. Enfermagem Revista, 15(3).

Weffort, V. R. S. (2019). Obesidade na infância e adolescência: Manual de Orientação (3rd ed.). SBP. 